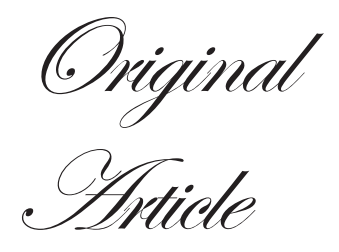

\title{
Insulin resistance and metabolic syndrome in non- obese Indian patients with non-alcoholic fatty liver disease
}

\author{
Ganesh Bhat ${ }^{1}$, Chalamalasetty Sreenivasa Baba ${ }^{1}$, Amaresh Pandey $^{1}$, \\ Neeraj Kumari ${ }^{2}$, Gourdas Choudhuri ${ }^{1}$
}

\section{ABSTRACT}

Department of Gastroenterology 1

Department of Pathology ${ }^{2}$

Sanjay Gandhi Postgraduate Institute

of Medical Sciences, Lucknow

Correspondence

Dr. G Choudhuri

Email: choudhuri.gour@gmail.com
Background: Insulin resistance has been recognized as a major factor in the development of non-alcoholic fatty liver disease (NAFLD). The association between insulin resistance and

NAFLD, as a risk factor independent of obesity has been less well established. This study aims to determine presence of insulin resistance and components of metabolic syndrome in non-obese patients with NAFLD.

Methods: 150 patients (mean age $42.25 \pm 10.50 \mathrm{y}$; 115 (76\%) male, 35 (24\%) female) diagnosed with NAFLD participated in the study. We measured body mass index (BMI), waist circumference (WC), waist hip ratio (WHR), fasting lipid profile, fasting glucose, fasting insulin, and liver function. Insulin resistance was calculated using the homeostasis model of assessment (HOMA) formula. Insulin resistance was arbitrarily considered altered when it was $>1.64$.

Results: 120 (80\%) of the 150 patients were obese (BMI >23) according to the Asia Pacific criteria. $40(30 \%)$ had metabolic syndrome. $97.5 \%(117 / 120)$ had insulin resistance with mean HOMA-insulin resistance (IR) of 10.9 \pm 5.3 . Thirty (20\%) were non-obese; of these, 7 had central obesity (WC $>90 \mathrm{~cm}$ for men, $>80 \mathrm{~cm}$ for women). Twenty-three $(15.3 \%)$ patients were lean NAFLD with BMI 21.6 \pm 1.5 , WC 82.9 \pm 4.7 (BMI $<23, \mathrm{WC}<90 \mathrm{~cm}$ in men and $<80 \mathrm{~cm}$ in women) $80 \%$ of these 23 (18/23) had insulin resistance with mean HOMA-IR of 3.4 \pm .1.9. Only $4(17 \%)$ did not have any component of metabolic syndrome.

Conclusion: Insulin resistance often associated with metabolic syndrome is common and plays a key role amongst lean Indian patients with non-alcoholic fatty liver disease.

KEYWORDS: Lean NASH, central obesity, Fatty liver disease, overweight

\section{Introduction}

Nonalcoholic fatty liver disease (NAFLD) is increasingly recognized as a common cause of elevated serum aminotransferase levels in the western population and is an emerging problem in the Asia-Pacific region. ${ }^{1,2,3,4}$ NAFLD is part of the spectrum of clinicopathologic liver disease ranging from simple steatosis to steatohepatitis (NASH) and can potentially progress to cirrhosis and liver failure ${ }^{5}$ Recent studies suggest that NAFLD has an estimated prevalence of $4-24 \%$ in the general population and may be the primary cause of cryptogenic cirrhosis in $15-20 \%$ of patients. ${ }^{4,6,7}$ With emerging clinical and epidemiological evidence, most clinicians generally regard NAFLD as a part of metabolic syndrome. ${ }^{8}$ With the obesity pandemic, NAFLD is going to be increasingly encountered by the clinician. ${ }^{9}$

(C) Tropical Gastroenterology 2013 
Available clinical and epidemiologic data show a clear relationship between obesity, type 2 diabetes mellitus, hyperlipidemia and NAFLD. ${ }^{9}$ Despite this, recent studies have reported a significant proportion of NAFLD patients who were non-obese and who did not meet the minimum criteria for metabolic syndrome in the western population. ${ }^{10,11}$ Further some reports have suggested that Asian people have more central obesity when compared to the western population for a given BMI. $^{12}$

Although the association of insulin resistance in obese NAFLD patients is well known, its role in non-obese NAFLD patients has been less well established. ${ }^{13}$ This prospective study was performed to determine the presence of insulin resistance and components of metabolic syndrome in nonobese, non-diabetic patients with non-alcoholic fatty liver disease.

\section{Patients and Methods}

Patients

Patients attending the liver clinic at our center with a diagnosis of NAFLD were included in the study. The diagnosis of NAFLD was based on ultrasound evidence of fatty liver and persistent elevation of serum alanine aminotransferase (ALT) $>1.5$ times the upper limit. We excluded patients with other causes of chronic hepatitis including viral hepatitis, autoimmune hepatitis, cholestatic liver disease, hemochromatosis, Wilson disease and alcoholic liver disease (alcohol use $>20 \mathrm{~g} /$ day). We also excluded patients with clinical suspicion or ultrasound evidence of portal hypertension. In addition, we also excluded patients who were known diabetic or had impaired glucose tolerance. Informed consent was obtained from each patient. The study was approved by the institute ethics committee.

\section{Methods}

\section{Clinical and Anthropometric Data}

Detailed history including drug use, particularly oral contraceptives, corticosteroids and ATT, and clinical examination to look for any evidence of chronic liver disease were done during initial screening. Body mass index (BMI; kg/ $\mathrm{m}^{2}$ ) was calculated using Quetelet index formula upon recording weight to the nearest half-kilogram with light clothes and bare feet, and height standing bare feet. ${ }^{15}$ Waist circumference (WC) and hip circumference $(\mathrm{HC})$ in centimeters were measured at the level of the umbilicus and at the widest portion of buttocks, respectively. Waist-hip ratio (WHR) was calculated by dividing waist circumference by hip circumference. Increased WHR was defined as $>0.90$ in men and $>0.85$ in women. ${ }^{16}$

\section{Metabolic syndrome}

Metabolic syndrome was defined according to the National Cholesterol Education Program (NCEP) adult treatment panel III (ATP III) guidelines as the presence of any 3 of the following 5 components ${ }^{17}$ : (1) Waist circumference $>102 \mathrm{~cm}$ [men] and $>$ $88 \mathrm{~cm}$ [women], (2) Fasting triglycerides $>150 \mathrm{mg} / \mathrm{dL}$, (3) High density lipoprotein cholesterol $<40 \mathrm{mg} / \mathrm{dL}$ [men] and $<50 \mathrm{mg} /$ $\mathrm{dL}$ [women], (4) blood pressure $>130 / 85 \mathrm{~mm} \mathrm{Hg}$, (5) fasting glucose $>110 \mathrm{mg} / \mathrm{dL}$. The normal waist circumference of adult Indians is lower than Caucasians; hence we used a cut off of $>$ $90 \mathrm{~cm}$ [men] and $>80 \mathrm{~cm}$ [women] as described for Indians ${ }^{18}$.

\section{Biochemical tests and serological markers}

Plasma glucose, both at fasting and following ingestion of $75 \mathrm{~g}$ of glucose was measured with an automated analyzer using glucose oxidase and peroxidase method. Fasting lipid profile for total cholesterol, low-density lipoprotein (LDL) cholesterol, high-density lipoprotein (HDL) cholesterol, very low-density lipoprotein (VLDL) and triglycerides (TGL) was obtained (RA$\mathrm{XT}$ random access clinical chemistry analyzer, Bayer Diagnostics, Tarrytown, NY, USA). Serum iron studies, albumin, aspartate aminotransferase (AST) and alanine aminotransferase (ALT) were done on all patients at baseline.

HBsAg (Hepanostika, Biomerieux bv, Boxtel, NL, US) and anti-HCV (UBI, United Biochemicals Inc, Houppauge, NY, US) to exclude hepatitis $\mathrm{B}$ and $\mathrm{C}$ infection; and anti nuclear antibody (ANA), anti smooth muscle antibody (ASMA), anti mitochondrial antibody (AMA) and anti liver kidney microsomal antibody (ALKM) were measured in all patients to exclude any concomitant presence of autoimmune hepatitis. Patients less than 40 years of age also underwent slit lamp examination to rule out Wilson's disease. TTG test was done in non-obese NAFLD patients to rule out celiac disease.

\section{Insulin resistance}

Fasting samples of serum obtained after centrifugation were stored at $-70^{\circ} \mathrm{C}$ until assayed. Fasting insulin levels (mU/L) were done using radioimmunoassay (Diagnostic Products Corporation, Los Angeles, CA). The homeostasis model of 
assessment for insulin resistance (HOMA-IR) was calculated on the basis of fasting values of plasma glucose and insulin according to the HOMA model formula:

HOMA-IR = fasting insulin $\mathrm{x}$ fasting glucose, divided by $22.5^{19}$

As previously suggested, insulin resistance was arbitrarily considered altered when it was $>1.64 .^{20}$

\section{Ultrasonography}

Ultrasonogram (USG) was performed in fasting state for grading the extent of fatty liver and to look for evidence of portal hypertension. The severity of echogenicity was graded as: Grade 0: normal echogenicity; Grade 1: slight, diffuse increase in fine echoes in liver parenchyma with normal visualization of diaphragm and intrahepatic vessel borders; Grade 2: moderate, diffuse increase in fine echoes with slightly impaired visualization of intrahepatic vessels and diaphragm; Grade 3: marked increase in fine echoes with poor or non-visualization of the intrahepatic vessel borders, diaphragm, and posterior right lobe of the liver. ${ }^{21}$.

\section{Liver Biopsy}

Liver biopsy was done using Menghini's needle as an indoor procedure in those who consented. A single pathologist blinded to all clinical data scored biopsies using the Brunt's criteria for grading and staging of non-alcoholic steatohepatitis $^{22}$. Briefly, steatosis was graded as 0-3: grade 0 , no steatosis; grade $1,<33 \%$ of biopsy; grade $2,33 \%$ to $66 \%$; grade $3,>66 \%$ of biopsy. Necroinflammatory activity was graded 1-3 and fibrosis staged from 1-4: stage 1, zone 3 fibrosis, focally or extensively present; stage 2, zone 3 fibrosis with periportal fibrosis; stage 3, zone 3 fibrosis with bridging fibrosis; stage 4, cirrhosis.

\section{Study Design}

All patients were divided into 3 categories according to their BMI and Waist circumference.

1. Obese or overweight according to Asia-Pacific criteria According to BMI; < 18.5 - underweight, 18.6-22.9- normal, 23-24.9 - overweight, 25-29.9 - grade I obesity, > 30 - morbid obesity.

2. Normal BMI, but central obesity according to Asia-Pacific criteria: (BMI 18.6-22.9) WC $>90 \mathrm{~cm}$ in men, $\mathrm{WC}>80 \mathrm{~cm}$ in women.
3. Lean body weight according to Asia-Pacific criteria: BMI 18.6-22.9 and $\mathrm{WC}<90 \mathrm{~cm}$ in men, $<80 \mathrm{~cm}$ in women.

\section{Statistical Analysis}

All data have been expressed as mean \pm SD unless otherwise indicated. Two tailed Mann-Whitney U test and Student T test were applied for comparison of groups. Correlation between insulin resistance and waist circumference, BMI, ALT level was done using Pearson's correlation test. The association between insulin resistance and BMI, WC, ALT was done using Pearson's correlation coefficient. Differences were considered statistically significant at $\mathrm{p}<0.05$. All analyses were performed using SPSS 16.0 software (SPSS Inc., Chicago, IL, US).

\section{Results}

Of the 280 consecutive patients attending our specialist clinic, 150 non-diabetics, non-alcoholics were included in the study. Most of our patients had nonspecific symptoms like dyspepsia, right hypochondriac pain or had been referred to our clinic for further evaluation after incidental detection of fatty liver on USG and/or elevated transaminases on routine evaluation.

\section{Demography and baseline parameters}

The majority of our patients, 115 , were male $(76 \%)$, with mean age $42.2 \pm 8.2$ years. All patients were nonalcoholic and had negative viral markers. $40(27 \%)$ patients had grade 1 fatty liver, $80(53 \%)$ had grade 2 fatty liver and $30(20 \%)$ had grade 3 fatty liver on USG.

Demography and laboratory parameters of the four groups

$120(80 \%)$ of 150 patients were overweight/obese $\left(>23 \mathrm{~kg} / \mathrm{m}^{2}\right)$ (Group I) according to Asia Pacific criteria. 40 (30\%) of 120 patients had metabolic syndrome. They showed significant difference in anthropometric and insulin resistance from nonobese patients. (Table 1)

Seven $(5 \%)$ of 150 patients had central obesity according modified Indian criteria (Group 2). These patients had significantly lower insulin resistance level compared to obese patients. (Table 2)

Twenty-three patients (15\%) of 150 were lean NAFLD patients (normal BMI and WC) (Group 3). Eighteen (80\%) of 23 lean NAFLD patients had insulin resistance $>1.64$ compared 
to $97.5 \%(117 / 120)$ of obese patients. However, their insulin resistance was significantly lower compared to obese patients $3.4 \pm 1.3$ vs. $10.9+5.3(\mathrm{p}<0.001)$ (Table 2). Four $(17 \%)$ had metabolic syndrome. Only 2 patients (9\%) of 23 did not have any component of metabolic syndrome. (Table 3 )

Table 1: Comparison between obese and non-obese $\left(B M I<23 \mathrm{~kg} / \mathbf{m}^{2}\right)$

\begin{tabular}{lccc}
\hline Characteristic & $\begin{array}{c}\text { Obese } \\
(\mathbf{n = 1 2 0})\end{array}$ & $\begin{array}{c}\text { Non-obese } \\
(\mathbf{n}=\mathbf{3 0})\end{array}$ & p value* \\
\hline Age $(\mathrm{yrs})$ & $42.8 \pm 8.3$ & $39.9 \pm 7.4$ & $\mathrm{~ns}$ \\
BMI $\left(\mathrm{kg} / \mathrm{m}^{2}\right)$ & $27.7 \pm 3.3$ & $21.7 \pm 1.3$ & $<0.001$ \\
WC & $97.7 \pm 5.1$ & $84.9 \pm 5.0$ & 0.001 \\
Fasting glucose (mg/dL) & $88.1 \pm 17.3$ & $87.5 \pm 14.4$ & $\mathrm{~ns}$ \\
2 hr glucose (mg/dL) & $127.8 \pm 32.2$ & $125.5 \pm 31.8$ & $\mathrm{~ns}$ \\
ALT (U/L) & $82.9 \pm 45.6$ & $69.9 \pm 29.8$ & $\mathrm{~ns}$ \\
Total cholesterol (mg/dL) & $190.8 \pm 54.5$ & $177.7 \pm 36.9$ & $\mathrm{~ns}$ \\
Triglycerides (mg/dL) & $175.6 \pm 108.8$ & $185.6 \pm 114.4$ & $\mathrm{~ns}$ \\
LDL (mg/dL) & $124.2 \pm 42.3$ & $118.2 \pm 52.7$ & $\mathrm{~ns}$ \\
HDL (mg/dL) & $36.8 \pm 7.8$ & $38.0 \pm 8.4$ & $\mathrm{~ns}$ \\
HOMA-IR & $9.6 \pm 5.5$ & $3.5 \pm 1.4$ & 0.001 \\
\hline
\end{tabular}

*Student T- test

Table 2: Subgroup analysis of the anthropometric, aminotransferases and insulin resistance

\begin{tabular}{lcccc}
\hline Variable & $\begin{array}{c}\text { Group I } \\
(\mathbf{n = 1 2 0})\end{array}$ & $\begin{array}{c}\text { Group II } \\
(\mathbf{n}=\mathbf{7})\end{array}$ & $\begin{array}{c}\text { Group III } \\
(\mathbf{n = 2 3})\end{array}$ & p value* \\
\hline Age $(\mathrm{yrs})$ & $42.8 \pm 8.3$ & $44.7 \pm 7.9$ & $38.4 \pm 6.7$ & $\mathrm{~ns}$ \\
BMI $\left(\mathrm{kg} / \mathrm{m}^{2}\right)$ & $27.7 \pm 3.3$ & $22.2 \pm 0.9$ & $21.5 \pm 1.3$ & $<0.001$ \\
WC & $97.7 \pm 5.5$ & $91.1 \pm 2.9$ & $83.0 \pm 3.8$ & $<0.001$ \\
ALT (U/L) & $82.9 \pm 45.6$ & $73.5 \pm 12.0$ & $68.8 \pm 33.5$ & $\mathrm{~ns}$ \\
HOMA-IR & $9.6 \pm 5.5$ & $4.0 \pm 1.6$ & $3.4 \pm 1.3$ & $<0.001$ \\
\hline
\end{tabular}

*Mann-Whitney U test

Table 3: Components of metabolic syndrome seen in our patients n(\%) according to NCEP ATP III and waist modified by Indian criteria

\begin{tabular}{lcc}
\hline Components present & NCEPATPIII & Indian criteria \\
\hline None & $10(6)$ & $2(1)$ \\
1 & $59(39)$ & $33(22)$ \\
2 & $57(38)$ & $60(40)$ \\
3 & $20(14)$ & $45(30)$ \\
4 & $4(3)$ & $10(7)$ \\
\hline
\end{tabular}

Degree of insulin resistance increased in a graded manner as waist circumference increased between these groups. (Figure 1)

\section{Correlation between insulin resistance and waist circumference}

We found significant correlation between degree of insulin resistance and waist circumference in NAFLD patients. $(\mathrm{p}=$ $0.01, r_{p}=0.79$ ). We found significant correlation between insulin

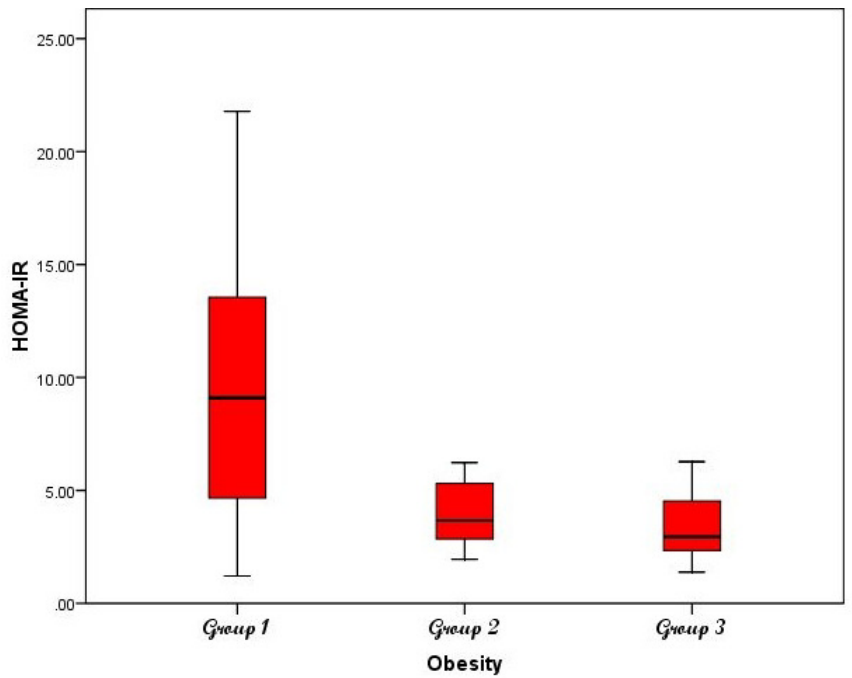

Figure 1: Box plot representation of insulin resistance in 3different group

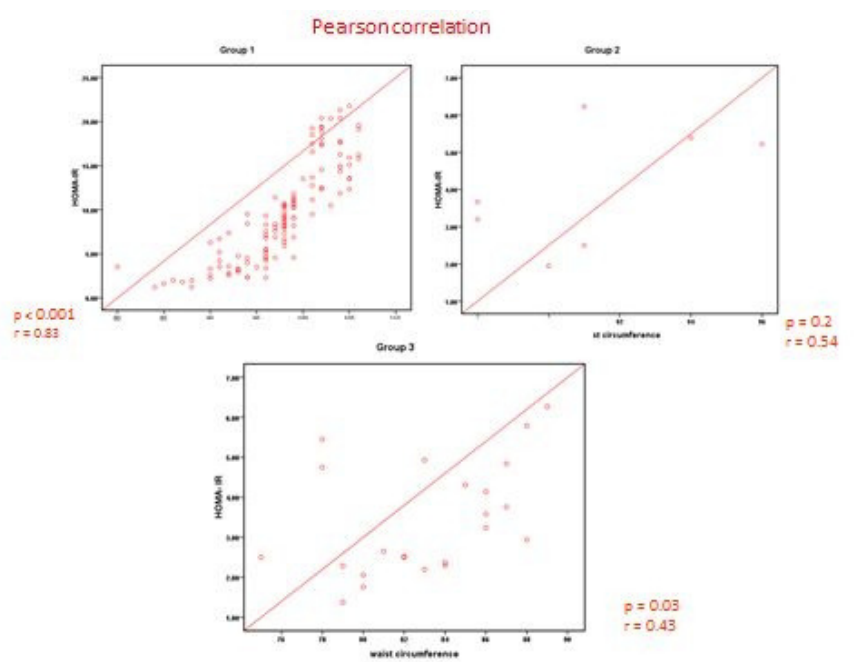

Figure 2: Correlation between insulin resistance and waist circumferencer

resistance and waist circumference in the obese group (Group 1), but not in the non-obese group (Figure 2). We did not document significant correlation between insulin resistance and BMI, ALT levels.

\section{Histopathology}

Forty-two (28\%) patients consented to liver biopsy and findings are given in Table 4. Macro-vesicular steatosis was present in all the biopsies (grade 1 in 15, grade 2 in 19, grade 3 in 8 ). Ballooning degeneration was seen in $10(23 \%)$ and glycogenated nuclei in 12 (28\%). Necro-inflammatory activity was present in all biopsies, the majority showed mild to moderated activity (grade 1 in 14, and grade 2 in 20); severe necro-inflammatoy activity was seen in 8 . There was no fibrosis 
Table 4: Histological characteristics of NASH on liver biopsy

\begin{tabular}{ll}
\hline Histological finding & $(\mathbf{n}=\mathbf{4 2})$ \\
\hline$\underline{\text { Steatosis }}$ & \\
- Grade 1 & 15 \\
- Grade 2 & 19 \\
- Grade 3 & 8 \\
Ballooning degeneration & 10 \\
Glycogenated nuclei & 12 \\
Necroinflammatory activity & \\
- Grade 1 & 14 \\
- Grade 2 & 20 \\
- Grade 3 & 8 \\
Staging Fibrosis & \\
- Stage 0 & 15 \\
- Stage 1 & 17 \\
- Stage 2 & 5 \\
- Stage 3 & 5 \\
\hline
\end{tabular}

in 15 patients and mild, grade 1 in 17 , and grade 2 in 5 . None of them showed cirrhotic changes while grade 3 fibrosis was seen in 5 patients.

\section{Discussion}

In our study a significant proportion of NAFLD patients ( 20\%) had normal BMI (lean body weight, BMI $<23 \mathrm{~kg} / \mathrm{m}^{2}$ ). In previous studies from India the prevalence of lean NAFLD (BMI $<23 \mathrm{~kg}$ / $\mathrm{m}^{2}$ ) was $\sim 12 \%$ in hospital-based data and $\sim 40 \%$ in populationbased data. ${ }^{4,23}$

Current evidence suggests that insulin resistance plays a central role in the pathogenesis of NAFLD, especially in obese patients with diabetes and related components of metabolic syndrome. ${ }^{24}$ The majority of lean NAFLD patients in our study had insulin resistance. In a recent study, Petersen, et al showed that prevalence of insulin resistance is higher in healthy lean Asian Indians compared to other ethnic groups. This increased prevalence in insulin resistance among Asian Indian men was associated with increased hepatic triglyceride content and plasma IL-6 concentration. ${ }^{25}$ Our result also suggests that insulin resistance plays a significant role in the pathogenesis of NAFLD in non-obese, non-diabetic patients.

In a previous study, insulin resistance was shown to be an independent risk factor for NAFLD in non-diabetic patients with normal BMI $\left(<25 \mathrm{~kg} / \mathrm{m}^{2}\right) .{ }^{26}$ But this study provided no details of prevalence of central obesity and proportion of lean patients (normal BMI, no central obesity). In our studies $20 \%$ patient had BMI $<23 \mathrm{~kg} / \mathrm{m}^{2}$, of which 23 patients (15\%) were lean $\left\{\right.$ normal BMI $\left(<23 \mathrm{~kg} / \mathrm{m}^{2}\right)$, no central obesity $\left.\}\right)$ according to Asia Pacific criteria. Four of them (17\%) had metabolic syndrome. Only 3 patients (13\%) of 23 did not have any component of metabolic syndrome when modified criteria of waist circumference for Asia-Pacific patients were used.

The most interesting observation made in our study was that 23 patients $(15 \%)$ were lean (according to the Asian criteria of BMI and WC). This highlights the need for lowering the cut off point for $\mathrm{WC}$ in these patients. A previous populationbased study suggested $\mathrm{WC}>90 \mathrm{~cm}$ for men and $>80 \mathrm{~cm}$ for women in patients without any risk factor for cardiovascular disease (Action I), whereas $\mathrm{WC}>80 \mathrm{~cm}$ for men and $\mathrm{WC}>72$ $\mathrm{cm}$ for women in patients with 1 or more risk factors for cardiovascular disease (Action panel=II), as cut off for central obesity in defining metabolic syndrome. ${ }^{18}$ Our study suggests that NAFLD should be considered a part of metabolic syndrome and WC criteria should be modified accordingly (in favor of Action panel=II).

In our study, the degree of insulin resistance correlated with waist circumference and not with BMI (Figure 1). This again stresses that insulin resistance correlates better with visceral obesity than with total body fat. Two previous studies have shown that lean NAFLD patients have visceral adipose tissue volume comparable to overweight and obese patients. ${ }^{4,27}$

The majority of our patients had milder histological changes and none had cirrhosis. Previous studies have shown that Indian patients tend to have milder histological disease compared to west. ${ }^{23,27,28}$ In a previous study the degree of steatosis was found to correlate with the degree of obesity; obese patients had severe liver disease (higher NASH score) compared with lean NAFLD patients. ${ }^{29}$ In our study as only 2 lean NAFLD patients underwent liver biopsy; we found no correlation between WC, Insulin resistance and degree of hepatic steatosis. In addition to this, the role of gene polymorphism of apolipoprotein C3 (APOC3) and patalin- like phospholipase domain-containing protein 3 (PNPLA3) in predicting severity of disease in lean NAFLD patients needs further study. ${ }^{30}$

The other highlight of our study is steady increase in insulin resistance proportional to graded increase in WC (central obesity), again indicating that central obesity rather than BMI correlates with degree of insulin resistance. This implies lean NAFLD patients may be in the initial phases of metabolic syndrome, and increasing WC and eventually BMI result from increasing obesity and its complications amongst these patients. Recent evidence suggests that early adoption of life style changes can prevent development of type 2 diabetes. ${ }^{31}$ As type 2 diabetes is the ultimate outcome of severe insulin resistance and NAFLD is another consequence of insulin 
resistance, adaptation of life style at this stage (lean NAFLD) may prevent progression to NASH and to irreversible stage of liver fibrosis. However this needs to be analysed in a prospective study.

To conclude, a significant proportion of NAFLD Indian patients are lean and this emphasizes the need for further modification of central obesity criteria. Insulin resistance associated with components of metabolic syndrome plays a key role in non-obese non-diabetic patients.

\section{References}

1. Angulo P. Nonalcoholic fatty liver disease. $N$ Engl J Med. 2002;346:1221-31.

2. Neuschwander-Tetri BA, Caldwell SH. Nonalcoholic steatohepatitis: summary of an AASLD Single Topic Conference. Hepatology. 2003;37:1202-19.

3. Farrell GC, Chitturi S, Lau GKK, Solono JD; Asia-Pacific Working Party on NAFLD. Guidelines for the assessment and management of non-alcoholic fatty liver disease in the Asia Pacific region: Executive summary. J Gastroenerol Hepatol, 2007;22:775-7.

4. Das K, Das K, Mukherjee PS, Ghosh A, Ghosh S, Mridha AR, et al. Nonobese population in a developing country has a high prevalence of nonalcoholic fatty liver and significant liver disease. Hepatology. 2010;51:1593-602.

5. Farrell GC, Larter CZ. Nonalcoholic fatty liver disease: from steatosis to cirrhosis. Hepatology. 2006;43:S99-112.

6. Clark JM. The epidemiology of nonalcoholic fatty liver disease in adults. J Clin Gastroenterol. 2006;40:S5-10.

7. Ayata G, Gordon FD, Lewis WD, Pomfret E, Pomposelli J, Jenkins RL et al. Cryptogenic cirrhosis: clinicopathologic findings at and after liver transplantation. Human Pathol. 2002;33:1098-104.

8. Marchesini G, Brizi M, Bianchi G, Tomassetti S, Bugianesi E, Lenzi M, et al. Nonalcoholic fatty liver disease: A feature of the metabolic syndrome. Diabetes. 2001;50:1844-50.

9. Hedley AA, Ogden CL, Johnson CL, Carroll MD, Curtin LF, Flegal KM. Prevalence of overweight and obesity among US children, adolescents, and adults, 1999-2002. JAMA. 2004;291:2847-50.

10. Bellentani S, Saccoccio G, Masutti F, Croce LS, Brandi G, Sasso F, et al. Prevalence of and risk factors for hepatic steatosis in Northern Italy. Ann Intern Med. 2000;132: 112-7.

11. Ruhl CE, Everhart JE. Determinants of the association of overweight with elevated serum aminotransferase activity in the United States. Gastroenterology. 2003;124:71-9.

12. Marchesini G, Bugianesi E, Forlani G, Cerrelli F, Lenzi M, Manini $\mathrm{R}$, et al. Nonalcoholic fatty liver, steatohepatitis and metabolic syndrome. Hepatology. 2003;37:917-23.

13. Whincup PH, Gilg JA, Papacosta, O, Seymour C, Miller GJ, Alberti KG, et al. Early evidence of ethnic differences in cardiovascular risk: cross sectional comparison of British South Asian and white children. BMJ. 2002;324:635-8.
14. Pagano G, Pacini G, Musso G, Gambino R, Mecca F, Depetris $\mathrm{N}$, et al. Nonalcoholic steatohepatitis, insulin resistance, and metabolic syndrome: further evidence for an etiologic association. Hepatology. 2002;35:367-72.

15. Willet WC, Dietz WH, Colditz GA. Guidelines for healthy weight. N Engl J Med. 1999;341:427-34.

16. World Health Organization. Definition, diagnosis and classification of diabetes mellitus and its complications - Part 1: Diagnosis and classification of diabetes mellitus. Geneva: World Health Organization; 1999:20-1.

17. Expert Panel on Detection Evaluation and Treatment of High Blood Cholesterol in Adults. Executive summary of the third report of the National Cholesterol Education Program (NCEP) expert panel on detection, evaluation, and treatment of high blood cholesterol in adults (Adult Treatment Panel III). JAMA. 2001;285:2486-97.

18. Misra A, Vikram NK, Gupta R, Pandey RM, Wasir JS, Gupta VP. Waist circumference cutoff points and action levels for Asian Indians for identification of abdomen adiposity. Int J Obes (Lond). 2006;30:106-11.

19. Banora E, Targher G, Alberiche M, Bonadonna RC, Saggiani F, Zenere MB, et al. Homeostasis model assessment closely mirrors the glucose clamp technique in the assessment of insulin sensitivity: studies in subjects with various degrees of glucose tolerance and insulin sensitivity. Diabetes Care. 2000;23:57-63.

20. Chitturi S, Abeygunasekera S, Farrell GC, Holmes-Walker J, Hui JM, Fung C, et al. NASH and insulin resistance: Insulin hypersecretion and specific association with the insulin resistance syndrome. Hepatology. 2002;35:373-9.

21. Sanyal AJ. American Gastroenterological Association AGA technical review on nonalcoholic fatty liver disease. Gastroenterology. 2002;123:1705-25

22. Brunt EM, Janney CG, Di Bisceglie AM, Neuschwander-Tetri BA, Bacon BR. Non-alcoholic steatohepatitis: A proposal for grading and staging the histological lesions. Am J Gastroenterol. 1999;94:2467-74.

23. Duseja A, Das A, Das R, Dhiman RK, Chawla Y, Bhansali Y, Kalra N. The Clinicopathological Profile on Indian Patients with Nonalcoholic Fatty Liver Disease (NAFLD) is different from that in the West. Dig Dis Sci. 2007;52:2368-74.

24. International Obesity Taskforce. The Asia-Pacific Perspective: Redefining obesity and its treatment. Sydney: Health Communications Australia. 2000:1-56

25. Petersen KF, Dufour S, Feng J, Befroy D, Dziura J, Dalla Man $\mathrm{CD}$, et al. Incresed prevalence of insulin resistance and nonalcoholic fatty liver disease in Asian- Indian men. PNAS. 2006;108;18273-7.

26. Park SH, Kim BI, Yun JW, Kim JW, Park DI, Cho YK, et al. Insulin resistance and $\mathrm{C}$-reactive protein as independent risk factors for non-alcoholic fatty liver disease in non-obese Asian men. J Gastroenterol Hepatol. 2004;19:694-8.

27. Madan K, Batra Y, Gupta SD, Chander B, Rajan KD, Tewatia MS, et al. Non- alcoholic fatty liver disease may not be a severe disease at presentation among Asian Indians. World $J$ Gastroenterol. 2006;12:3400-5.

28. Duseja A, Das A, Dhiman RK, Chawla YK, Das R, Bhadada S, et al. Indian patients with nonalcoholic fatty liver disease presenting 
with raised transaminases are different at presentation. World $J$ Gastroenterol. 2007;13:649-50.

29. Choudhary NS, Duseja A, Kalra N, Das A, Dhiman RK, Chawla YK. Correlation of adipose tissue with liver histology in Asian Indian patients with Nonalcoholic fatty liver disease (NAFLD). Ann Hepatol. 2012;11:478-86.

30. Duseja A, Aggarwal R. APOC3 and PNPLA3 in Non-alcoholic fatty liver disease: Need to clear the air. J Gastroenterol Hepatol. 2012;27:848-51.

31. Knowler WC, Barrett-Conor E, Fowler SE, Hamman RF, Lachin JM, Walker EA et al. Reduction in incidence of type 2 diabetes with lifestyle intervention or metformin. $N$ Eng J Med. 2002;346:393-403. 